\title{
Metodologias da Engenharia de Requisitos: identificação e avaliação das orientações*
}

\author{
Requirements Engineering Methodologies: \\ identification and evaluation of orientations
}

Roberto Avila Paldês ${ }^{1}$ Angélica Toffano Seidel Calazans ${ }^{2}$ Tiago Pincowscy Cardoso $\mathrm{Maia}^{3}$ Isabel Sofia Sousa Brito ${ }^{4}$ Ari Melo Mariano 5

Recebido em: 07/01/2016.

Aprovado em: 14/09/2016.

1 Mestre em Educação pela Universidade Católica de Brasília. Graduado e Especialista o em Análise e Desenvolvimento de Sistemas.

2 Doutora em em Ciência da Informação pela Universidade de Brasilia. Mestre em Gestão de conhecimento e TI pela Universidade Católica de Brasília. Professora da graduação superior em Administração e em Análise de Sistemas, bem como da pós-graduação em Engenharia de Requisitos do UniCEUB.

3 Especialista em Análise de Requisitos pelo UniCEUB.

4 Professor of Computer Science, Polytechnic Institute of Beja. Doutora em Informática pela Universidade Nova de Lisboa.

Faculdade de Agronomia e Medicina Veterinária, Brasília. Pós Doutor em Negócios Internacionais e Metodologia Cientifica. Doutor em Administração pela UFBA, Doutor em Negócios Internacionais pela Universidade de Sevilla, Espanha, Mestrado em gestão e direção de empresas pela Universidade de Sevilla-España, MBA em Direito do Consumidor Europeu pela Universidade de Sevilla, Graduado em Marketing pelo Centro Universitário da Bahia.

\section{Resumo}

Este artigo parte de uma revisão sistemática para avaliar as abordagens existentes no domínio da Engenharia de Requisitos (ER) e suas implementações. O objetivo foi identificar quais as metodologias da ER são mais citadas no meio acadêmico, identificando as orientações existentes e as que despertam mais interesse no meio acadêmico e/ou na indústria. Foram utilizadas as bases da Web of Science e o Google Acadêmico, considerando-se o período de 20 anos (1994 a 2015). Com base na definição da metodologia da pesquisa, foram elaboradas questões para avaliar a qualidade e adequação dos trabalhos ao contexto da investigação. Foram analisados 184 artigos, sendo identificadas as metodologias Goal, Scenario e Object-oriented como as mais representativas em quantitativo de artigos e citações. Identificou-se, também, que metade dos trabalhos possui experimentação e que, quando ela ocorre, prevalece no contexto industrial. O trabalho complementa a visão de trabalhos anteriores que abordam as técnicas usadas em fases específicas da ER.

Palavras-chave: Engenharia de requisitos. Revisão sistemática da literatura. Metodologias.

\section{Abstract}

This article is based on a systematic review to evaluate existing approaches in the field of Requirements Engineering (RE) and their implementations. The objective was to identify the methodologies of ER are most cited in academia, identifying existing guidelines and that arouse more interest in academia and / or industry. The bases Web of Science and Google Scholar were used, considering the 20-year period (1994-2015). From the definition of research methodology, questions were designed to assess the quality and appropriateness of the work to the research context. It was analyzed 184 articles, identified methodologies Goal, Scenario and Object-oriented as the most representative in quantity of articles and citations. It is also identified that about half of work has experimentation and they occurs in industrial environment. The work complements the vision of previous works that address the techniques used at specific stages of the ER.

Keywords: Requirements engineering. Systematic literature reviews. Methodologies. 


\section{Introdução}

Umas das maiores dificuldades no processo de construção de um sistema é entender as necessidades dos stakeholders e o que eles esperam do sistema. Como tornar aquilo que é imaginado em um sistema funcional que atenda todas as exigências do negócio ou da organização? Esse processo faz parte da Engenharia de Requisitos (ER), a fase inicial da construção de um sistema. As atividades da ER se desenvolvem em torno de entender o problema, descrevê-lo, adequadamente, e assegurar que isso foi realizado corretamente (ANTONELLI; OLIVEROS, 2015). Além disso, ela, também, engloba a atividade de gerenciar esses requisitos de forma a manter a sua coerência e integridade, com o projeto, após as mudanças.

A Engenharia de Requisitos (IEEE, 1998) compreende os processos de obtenção, refinamento e verificação das necessidades do usuário, por meio do uso de técnicas sistemáticas e repetíveis a serem usadas para assegurar que os requisitos do software sejam completos, consistentes, relevantes e que atendam às necessidades das partes interessadas no sistema (stakeholders). Existem quatro atividades importantes no processo da engenharia de requisitos: estudo de viabilidade; elicitação e análise dos requisitos; especificação dos requisitos e validação dos requisitos (SOMMERVILLE; SAWYER, 1997).

Todo esse processo, que envolve a comunicação entre os stakeholders e os desenvolvedores, é bastante complexo e apresenta muitos desafios e riscos para os desenvolvedores e para o sistema a ser construído, pois os requisitos são a base para essa construção. É nesse contexto que vários métodos, técnicas e metodologias foram propostos nos últimos anos objetivando mitigar ou superar essas barreiras. Algumas dessas metodologias foram somente propostas, enquanto outras foram implementadas em ambientes acadêmicos e industriais.

Considerando-se o exposto e motivados pelos mais de 20 anos da existência da área de domínio da Engenharia de Requisitos, este trabalho tem como objetivo apresentar uma revisão sistemática da literatura, com o intuito de pesquisar as metodologias da ER implementadas no meio acadêmico ou na indústria.

Diferencia-se o estudo por ter uma abordagem mais ampla do que as técnicas ou ferramentas disponíveis para uma determinada atividade da ER. O termo metodologia é entendido como "um conjunto de ferramentas, técnicas, métodos, princípios e regras organizados de forma clara, lógica e sistemática, para uso como guia, e uma descrição passo a passo de como se alcançar alguma coisa" (PEREZ-WILSON, 1999).

O trabalho está organizado da seguinte maneira. A Seção 2 descreve o método de pesquisa utilizado, incluindo etapas da pesquisa, as strings de busca e as limitações. Na Seção 3, são apresentados os resultados e, por último, a Seção 4 expõe as conclusões sobre o trabalho.

\section{Metodologia da pesquisa}

Para atender o objetivo deste trabalho, foi realizada uma revisão sistemática de literatura, pois ela pode fornecer uma visão geral da área de requisitos para avaliar a quantidade de dados existentes sobre um tópico de interesse e as tendências para orientar futuros estudos. A presente revisão sistemática foi planejada e executada de acordo com os procedimentos estabelecidos nos trabalhos de Kitchenham (2007) e Biolchini et al. (2005).

$\mathrm{Na}$ sequência, se apresenta o escopo da investigação, as perguntas de pesquisa e o método da pesquisa, que incluem o planejamento da investigação do processo de pesquisa, bem como os critérios de inclusão e exclusão da coleta de dados e sua análise. O protocolo de revisão sistemática foi concebido e conduzido por um aluno pesquisador da pós-graduação e validado por dois professores pesquisadores que revisaram o protocolo, validaram a inclusão e exclusão de artigos e discutiram os resultados da avaliação.

\subsection{Escopo da investigação}

O escopo desta pesquisa envolve a identificação e a análise dos artigos publicados sobre o tema à procura das metodologias adotadas na Engenharia de Requisitos e a sua implementação no meio acadêmico ou indústria, utilizando o método de revisão sistemática de literatura (KITCHENHAM, 2007; BIOLCHINI et al., 2005). Os métodos e processos foram adotados como um caminho para identificar as metodologias, uma vez que eles revelam as diversas formas ordenadas de proceder na produção de um resultado. A metodologia é mais ampla, pois corresponde à aplicação do método, por meio de processos e técnicas, que garantem a legitimidade dos resultados obtidos (BARROS; LEHFELD, 2007). A metodologia pode ser o produto de abordagens propostas que tiveram sua eficácia testada e aprovada. Nesse sentido, as abordagens também foram entendidas como 
caminhos para identificar os estilos que orientam e os principais arcabouços teóricos adotados na Engenharia de Requisitos.

\subsection{Perguntas da pesquisa}

Para atender ao objetivo da pesquisa, foram definidas as seguintes questões do tipo descritivas, voltadas para a descrição das metodologias adotadas, buscando avaliar seu impacto e aceitação:

PP01 - Quais são as metodologias de ER mais citadas?

PP02 - Em relação às metodologias de ER mais citadas, quais as que foram mais implementadas na indústria ou no meio acadêmico?

PP03 - Considerando-se esses fatores, quais são as metodologias de ER que estão em crescimento no meio acadêmico?

\subsection{Método de Pesquisa}

A revisão foi realizada no período de fevereiro a julho de 2015 e as bases de dados utilizadas foram a Thomson Reuter Web of Science e o Google Acadêmico, que contem publicações no período de 1996 à 2014. A Web of Science (2015) é conhecida internacionalmente como uma das melhores e mais completas (GARCIA; RAMIREZ, 2004). Por se tratar de uma fonte mundial e com uma gama de autores e artigos científicos publicados, o Google Acadêmico foi utilizado para pesquisas, combinado com o software Publish or Perish (HARZING, 2015), um programa simples e gratuito que funciona como uma interface de busca do Google Acadêmico (MUGNAINI; STREHL, 2008). Não foram utilizadas outras bases bastante difundidas no meio acadêmico, como o IEE Explore e a ACM Digital, por terem seus periódicos da ER já indexadas na base da Thomson Reuter e para evitar duplicação nas contagens, evitando assim que um mesmo trabalho seja contado mais de uma vez. O Google Acadêmico já apresenta critérios diferenciados para a indexação, o que pode permitir a complementação de dados, com pequeno risco de avaliação repetida.

Assim, a revisão foi dividida em 2 etapas. A primeira etapa corresponde à identificação das metodologias citadas e a segunda etapa corresponde à investigação da relevância de cada uma delas.

\subsubsection{Primeira etapa}

Esta etapa teve como objetivo identificar as me- todologias existentes relacionadas à engenharia de requisitos. Assim, foi utilizado o termo "Requirements Engineering" e as palavras-chaves para identificar todas as possíveis abordagens sobre ER nas bases de dados consideradas. As palavras-chaves usadas nesse primeiro modulo da pesquisa foram: ("Methodology " OR "Method") OR ("Process").Os resultados foram refinados utilizando os seguintes tópicos: ("Science Technology” AND ("Computer Science" OR "Engineering") And ("Scientific Techno-

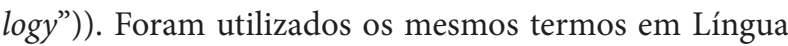
Portuguesa e em Língua Espanhola.

\subsubsection{Critérios de seleção e classificação}

Uma vez que os estudos primários potencialmente relevantes foram obtidos, eles precisaram ser avaliados para identificar sua relevância. Assim, com base em Kitchenham (2007) e Garcia e Ramirez (2004), em relação aos questionamentos de pesquisa, foram definidos os critérios de inclusão e exclusão que estão apresentados na Tabela 1.

Tabela 1 - Critérios de inclusão e exclusão da pesquisa

\begin{tabular}{ll}
\hline \multicolumn{1}{c}{ Critérios de inclusão } & \multicolumn{1}{c}{ Critérios de exclusão } \\
\hline & •Trabalhos que não mencionam ER; \\
- Trabalho publicado e disponível para & - Trabalhos duplicados; \\
que a comunidade em geral faça a sua & - Trabalhos com 1 ou 0 citações; \\
leitura; & - Trabalhos informais e apresentações; \\
- Trabalhos originais nas línguas: inglês, & - Qualquer trabalho com data anterior a 1994 \\
português e espanhol. & (20 anos); \\
& - Não estar disponível para leitura.
\end{tabular}

Fonte: do Autor

Além disso, todos os artigos com título ou palavras-chave que possam apresentar uma nova abordagem da ER tiveram seus resumos, introduções e conclusões manualmente checados e analisados para identificar a sua relevância quanto à pesquisa. Para seleção e classificação dos trabalhos em abordagens, foram adequadas as seguintes questões das propostas de Dyba e Dingsoyr (2008) e de Oliveira et al. (2013), sendo aplicada uma classificação em pontos:

O objetivo do estudo foi claramente definido? (Sim - 1, Não - 0);

A abordagem proposta foi claramente definida? (Sim - 1, Não - 0);

O estudo aborda as quatro principais etapas da ER (elicitação, análise, especificação e validação) ou qualquer variação dessas etapas? (Sim -1, Não - 0)

Para todos os trabalhos que receberam 3 pontos, foram definidas as suas classificações de metodologia, ou, em relação à inexistência de uma classificação, foi criada uma. 


\subsubsection{Extração e análise de dados}

Como foi referido anteriormente, os resultados obtidos na primeira etapa tinham como objetivo identificar as abordagens/métodos existentes relacionadas(os) à Engenharia de requisitos, ou seja, questão PP01. Na base da Web of Science, foram identificados, em Língua Inglesa, 522 artigos, aplicado o critério de exclusão de quantidade de citações (acima de 2 citações, inclusive) esse número foi para 334 artigos com um total de 5192 citações. Com relação à Língua Portuguesa, foram encontrados 4 artigos e, aplicado o mesmo critério de exclusão, esse número foi reduzido para 2 artigos com total de 6 citações. Não foram encontrados artigos em Língua Espanhola, o que pode estar relacionado com os critérios de indexação da base e com od termos selecionados em relação aos preferidos pelos autores em Língua Espanhola.

$\mathrm{Na}$ base do Google Acadêmico, foram identificados, em Língua Inglesa, 1000 artigos na base (número máximo que a ferramenta utilizada apresenta). Aplicando-se o critério de exclusão de quantidade de citações (acima de 2 citações, inclusive), esse número foi para 771 artigos com um total de 32817 citações. Com relação à Língua Portuguesa, foram encontrados 30 artigos e, após a aplicação do mesmo critério de exclusão, esse número foi reduzido para 13 artigos com total de 99 citações. Com relação a Língua Espanhola, foram encontrados 20 artigos e, aplicado o mesmo critério de exclusão, esse número foi reduzido para 12 artigos com total de 79 citações.

A síntese dos resultados da extração inicial dos dados na etapa 1 pode ser visualizada na Tabela 2. Todos os dados dos artigos (título, abstract, autores, ano, palavras chaves, qtd citações, classificação do trabalho, base de dados ou revista) foram consolidados em planilhas excel para facilitar a pesquisa nas fases seguintes. Tabela 2 - Quantidade de artigos selecionados

\begin{tabular}{l|l|c|c}
\multicolumn{1}{c|}{ Língua } & \multicolumn{1}{|c|}{ Critério } & Web of Science & Google Acadêmico \\
\hline \multirow{3}{*}{ Inglês } & identificados & 522 & 1000 \\
& selecionados & 344 & 771 \\
& citações & 5192 & 32817 \\
\hline \multirow{3}{*}{ Português } & identificados & 4 & 30 \\
& selecionados & 2 & 13 \\
& citações & 6 & 99 \\
\hline \multirow{3}{*}{ Espanhol } & identificados & 0 & 20 \\
& selecionados & 0 & 12 \\
& citações & 0 & 79 \\
\hline
\end{tabular}

Fonte: do Autor
A tabela 3 apresenta o quantitativo total de trabalhos, considerando-se as duas bases (Web of Science e Google Acadêmico) identificados por metodologia nessa primeira etapa. Estão listados o quantitativo de artigos e a quantidade de citações. Considerando os objetivos da pesquisa foram selecionadas e classificadas as metodologias e ou processos (abordagens) que tivessem:

Acima do total de 5 citações, considerando-se o total de citações de todos os trabalhos identificados para a metodologia ou processo proposto ou adaptado;

Mais de um trabalho utilizando ou adaptando a metodologia ou processo proposto.

Conforme pode ser constatado na Tabela 3, o quantitativo de citações dos trabalhos selecionados (8961) corresponde a aproximadamente $23 \%$ das citações totais do resultado obtido de citações (38193). Como pode ser observado, na Tabela 3,80, 80\% das citações correspondem as abordagens Goal, Scenario-based e Object-Oriented e isso corresponde a aproximadamente $62 \%$ dos trabalhos selecionados (184).

Tabela 3 - Total de artigos identificados por metodologia

\begin{tabular}{lcccc}
\hline \multicolumn{1}{c}{ Metodologia } & $\begin{array}{c}\text { Quantidade } \\
\text { de artigos }\end{array}$ & $\%$ & $\begin{array}{c}\text { Quantidade } \\
\text { de Citaçôes }\end{array}$ & $\%$ \\
\hline $\begin{array}{l}\text { Goal-Based ER Method or } \\
\text { Goal Oriented Requirements }\end{array}$ & 36 & 19,57 & 3509 & 39,16 \\
\hline $\begin{array}{l}\text { Scenario-Based ER method } \\
\text { Object-oriented approach or }\end{array}$ & 49 & 26,63 & 2705 & 30,19 \\
$\begin{array}{l}\text { Object Oriented ER or } \\
\text { Use case approach }\end{array}$ & 30 & 16,30 & 1030 & 11,49 \\
\hline $\begin{array}{l}\text { Viewpoint-oriented requirements } \\
\text { definition }\end{array}$ & 8 & 4,35 & 538 & 6,00 \\
\hline $\begin{array}{l}\text { Aspect oriented } \\
\text { Business Process Oriented ER }\end{array}$ & 3 & 1,63 & 415 & 4,63 \\
\hline $\begin{array}{l}\text { Process or Business-0bject Oriented } \\
\text { Requirements Analysis }\end{array}$ & 24 & 13,04 & 298 & 3,33 \\
\hline Agile Software Requirements & 11 & 5,98 & 211 & 2,35 \\
\hline Service Oriented Requirements & 17 & 9,24 & 176 & 1,96 \\
\hline Agent-Based or Agent-Oriented & 6 & 3,26 & 79 & 0,88 \\
\hline \begin{tabular}{l} 
Total \\
\hline Fonte: do Autor
\end{tabular} & 184 & & 8961 & \\
\hline
\end{tabular}

As Figuras de 1 até 6 apresentam a evolução dessas três primeiras metodologias pelo período da pesquisa (últimos 20 anos). Aquela que obteve maior número de citações (Goal) tem apresentado um quantitativo estável de citações durante os últimos anos. Isso pode representar um interesse constante no meio acadêmico pela abordagem e seu estudo. 
Figura 1 - Evolução de trabalhos Goal

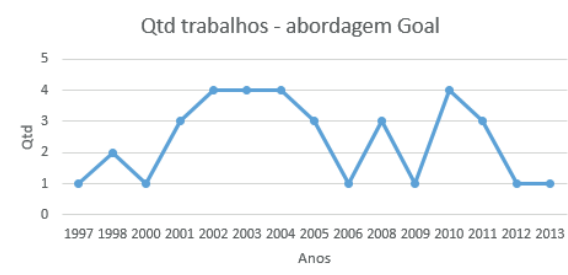

Fonte: do Autor

Figura 2 - Evolução de citações Goal Qtd citações - abordagem Goal

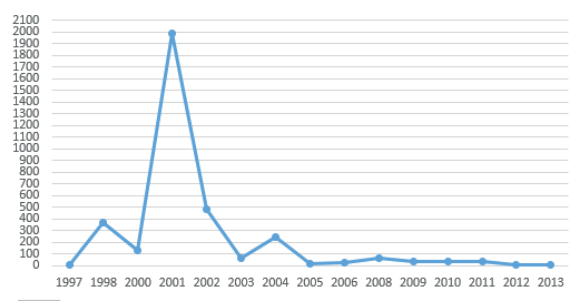

Fonte: do Autor

Figura 3 - Evolução de trabalhos Scenario Qtd trabalhos - abordagem Scenario

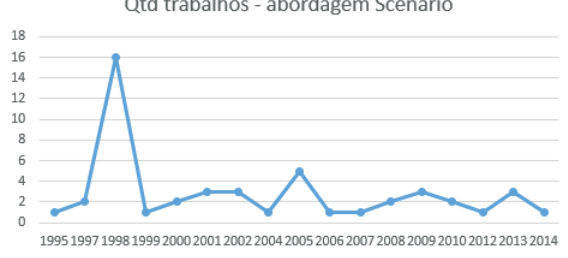

Fonte: do Autor

Figura 4 - Evolução de trabalhos Object oriented Qtd trabalhos - abordagem Object-oriented

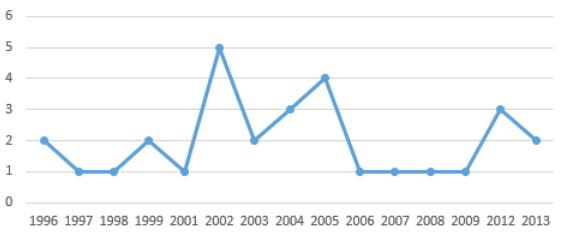

Fonte: do Autor

Figura 5 - Evolução de citações Scenario

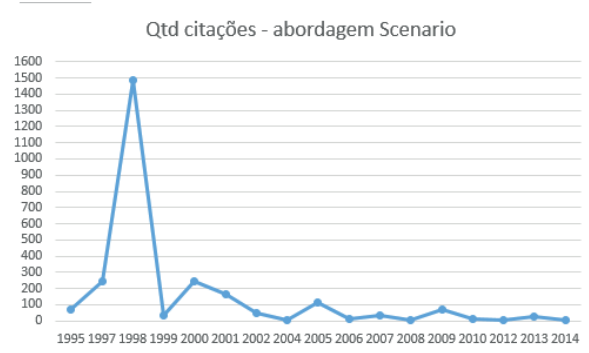

Fonte: do Autor

Figura 6 - Evolução de citações Object oriented Qtd citações - abordagem Object-oriented

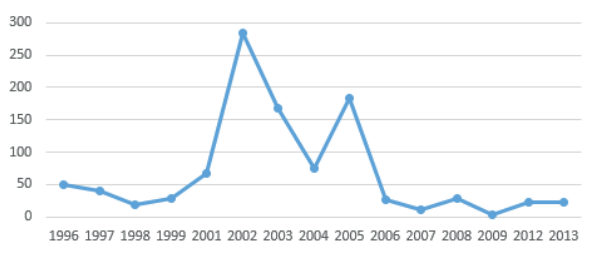

Fonte: do Autor

\subsubsection{Segunda etapa}

A segunda etapa da revisão seguiu critérios de avaliação da qualidade dos trabalhos conforme sugere Garcia e Ramirez (2004).

Considerando-se cada metodologia identificada, averiguando sua relevância à esta pesquisa e responder a responder a seguinte questão:

PP02: em relação às metodologias de $\mathrm{RE}$ mais citadas, quais as que foram mais implementadas na indústria ou no meio acadêmico?

Foram utilizados os mesmos critérios de seleção e classificação citados na Tabela 1. Além disso todos os artigos selecionados foram lidos e, manualmente, verificados e analisados. Foram adequadas as seguintes questões das propostas de Dyba e Dingsoyr (2008) e de Oliveira et al. (2013).

- A metodologia ou adequação apresentada foi implementada? (Sim, Não)

- No meio acadêmico ou no mercado?

- Em quantos projetos?

- Os resultados apresentados são claramente discutidos? (Sim, Não)

- Os resultados apresentados estão baseados em evidências? (Sim, Sim - mas as evidências não foram apresentadas, Não)

- Qual(is) tipo(s) de estudo(s) prevalence(m)?

A extração e análise de dados foi conduzida utilizando uma planilha contendo os seguintes campos: identificador; título; autores; data da publicação; data da leitura; fonte; nome da metodologia, quantidade de citações, metodologia, tipos de estudo, implementação ou não, quantidade de projetos.

O resultado da segunda etapa, apresentado na Tabela 4, revela, parcialmente, os artigos mais citados por metodologia considerada que foram analisados conforme os critérios definidos. 
Tabela 4 - Metodologias de ER selecionadas

\begin{tabular}{|c|c|c|c|}
\hline Metodologias & Síntese & Referências & $\mathrm{Nr}$ de citações \\
\hline Goal & $\begin{array}{l}\text { A metodologia Goal, tem como objetivo capturar, em diferentes } \\
\text { níveis de abstração, os objetivos do sistema. Requisitos orientados } \\
\text { a metas utiliza os objetivos para a obtençãa, elaboração, } \\
\text { estruturação, especificação, analise, documentação e modelagem } \\
\text { dos requisitos (VAN LAMSWEERDE, 2001) }\end{array}$ & $\begin{array}{l}\text { Van Lamsweerde (2001) } \\
\text { Yu e Mylopoulos (1998) } \\
\text { Fuxman (2004) } \\
\text { Van Lamsweerde (2004) } \\
\text { Castro, Kolp e Mylopoulos (2002) } \\
\text { Kaiya, Horai e Saeki (2002) } \\
\text { Van Lamsweerde e Letier (2000) } \\
\text { Kavakli (2002) }\end{array}$ & $\begin{array}{l}1717 \\
256 \\
233 \\
213 \\
193 \\
175 \\
132 \\
117\end{array}$ \\
\hline Scenario & $\begin{array}{l}\text { Os cenários são definidos como fatos que descrevem um sistema } \\
\text { existente e seu ambiente, incluindo o comportamento dos agentes } \\
\text { e informações contexto suficientes para permitir a descoberta e } \\
\text { validação de requisitos de sistema. Cenários possibilitam apresentar } \\
\text { as possíveis maneiras de usar um sistema para realizar alguma } \\
\text { função desejada. Clientes e usuários preferem falar de cenários } \\
\text { concretos em vez de modelos abstratos (SUTCLIFEE e RYAN, 1998). }\end{array}$ & $\begin{array}{l}\text { Rolland (1998) } \\
\text { Jarke, Bui e Carroll (1998) } \\
\text { Leite et al. (1997) } \\
\text { Leite, Hadad e Kaplan (2000) } \\
\text { Rolland, Souveyet, Achour (1998) } \\
\text { Sutcliffe e Ryan (1998) } \\
\text { Allenby e Kelly (2001) } \\
\text { Sutcliffe et al. (1998) }\end{array}$ & $\begin{array}{l}286 \\
276 \\
234 \\
214 \\
176 \\
147 \\
133 \\
125\end{array}$ \\
\hline Object-Oriented ER & $\begin{array}{l}\text { A orientação a objetos é uma abordagem para encapsular } \\
\text { informações sobre o processo e do produto, bem como a } \\
\text { funcionalidade em um objeto de requisitos. O paradigma de } \\
\text { orientaçãa a objetos, com relação a requisitos, está diretamente } \\
\text { representado como objetos de primeira classe. A notação de um } \\
\text { "objeto de requisitos" é usado para representar o objeto domínio } \\
\text { do problema. Um objeto de primeira classe tem o apoio de todas as } \\
\text { características do paradigma orientado a objetos e não apresenta } \\
\text { qualquer diferença entre os objetos no modelo de domínio e } \\
\text { objetos de requisitos em geral (Kaindl, 2005). }\end{array}$ & $\begin{array}{c}\text { Fantechi et al. (2003) } \\
\text { Santander e Castro (2002) } \\
\text { Evermann e Wand (2005) }\end{array}$ & $\begin{array}{l}158 \\
122 \\
117\end{array}$ \\
\hline
\end{tabular}

Fonte: do Autor

\section{Análise e discussão dos resultados}

A análise e discussão dos resultados foi classificada pelos questionamentos da pesquisa para facilitar o entendimento.

\subsection{Metodologias de ER mais citadas}

Considerando-se as abordagens definidas, foram identificadas as três principais abordagens mais citadas: "Goal-Based RE Method" ou "Goal Oriented Requirements", Scenario e Object-oriented. Essa conclusão não nos surpreende considerando outros trabalhos (VALASKI et al., 2014).

A metodologia mais citada é a "Goal-Based RE Method" ou "Goal Oriented Requirements" com aproximadamente $20 \%$ do total de trabalhos analisados. Os resultados obtidos no estudo de Oliveira et a. (2013) identificaram que $42 \%$ do total estudado relacionava-se à essa metodologia.

Na metodologia Goal, foram identificados diferentes notações e framework relacionados ao contexto de orientação a objetivos. Grande parte dos trabalhos utiliza e/ou cita o modelo $\mathrm{i}^{\star}$, Tropos com $\mathrm{i}^{\star}$, AGORA, KAOS, GRL (CASTRO; KOLP; MYLOPOULOS, 2002; KAIYA; HORAI; SAEKI, 2002; FUXMAN et al, 2004; ESPADA; GOULÃO; ARAÚJO, 2011). Foram encontrados trabalhos que apresentam complementação da me- todologia Goal com Scenario (ROLLAND; SOUVEYET; ACHOUR, 1998), com casos de uso, entre outros.

A metodologia Scenario foi a com maior quantitativo de trabalhos, aproximadamente $27 \%$ dos resultados selecionados. Foram identificadas diferentes propostas englobando e/ou citando modelos, tais como, Crews-savre, SCRAM, ISRE (SUTCLIFEE; RYAN, 1998; MAIDEN et al., 1998; SUTCLIFFE; GAULT; MAIDEN, 2005).

Já a metodologia Object-oriented corresponde a, aproximadamente, $16 \%$ dos resultados obtidos. Cerca de $70 \%$ dos trabalhos referem-se à utilização/adaptação de casos de uso.

\subsection{Implementação na indústria ou no meio acadê- mico e tipo de estudo}

Aproximadamente, $44 \%$ dos trabalhos relacionados a metodologia Goal não apresenta implementação em nenhum contexto, nem acadêmico nem industrial. Em relação ao restante dos trabalhos, ou seja 56\%, estudo empírico, ilustrações e estudos de caso foram os métodos mais utilizados. A maioria desses estudos ocorreu em ambiente industrial, sendo somente $25 \%$ ocorreu meio acadêmico. Esses números divergem do apontado pela pesquisa de Oliveira et al. (2013) que citou que os estudos acadêmicos correspondem a aproximadamente $89 \%$. A diferença pode estar na amplitude das amostras: en- 
quanto que o atual direcionou-se para as metodologias, o trabalho citado (OLIVEIRA et al., 2013) inclui técnicas para fases específicas (elicitação, validação, verificação, modelagem).

Cerca de 55\% dos trabalhos relacionados a metodologia Scenario não apresenta implementação em nenhum contexto, nem acadêmico, nem industrial. Em relação ao restante dos trabalhos, ou seja, 45\% dos resultados referentes à metodologia Scenario, estudos de caso foram os mais utilizados (85\%) e, destes, $90 \%$ foram na indústria. A maior parte dos trabalhos apresentou um estudo de caso.

Nesse contexto, alguns trabalhos iniciais abordando Scenario tratam a metodologia Goal e sua adequação para scenario, prototipação e cenários (ROLLAND; SOUVEYET; ACHOUR, 1998; SUTCLIFFE; GAULT; MAIDEN, 2005).

Com relação à metodologia Object-oriented, cerca de 53\% dos trabalhos não apresenta implementação em nenhum contexto, nem acadêmico, nem industrial. Em relação aos $47 \%$ restantes, a maioria cita estudos de caso da indústria (normalmente 1 estudo de caso por trabalho) e simulações (com dados fictícios para apresentar o modelo proposto). Poucos são os estudos de caso implementados no meio acadêmico.

\subsection{Metodologias em crescimento}

Foi observado que o maior quantitativo de citações ocorre nos anos em que foram lançadas as metodologias, conforme pode ser observado nas Figuras de 1 a 6. A partir daí, observa-se que, para as três metodologias, há um decréscimo de citações. Isso permite inferir que esse assunto já foi muito discutido pelo mercado e que, atualmente, está consolidado, com poucos trabalhos sendo realizados nesse contexto. O baixo quantitativo de trabalhos com experimentações, permite inferir que ainda há espaço para realização de mais pesquisas no mercado com essas abordagens.

Foram analisadas as outras metodologias, apesar do menor quantitativo de citações, visando identificar se, nos últimos 5 anos, houve um crescimento ou decréscimo do interesse do meio acadêmico pela metodologia. Foi identificado que as metodologias: Business process, Agile e Service-Oriented, apesar do quantitativo de citações menor, tem obtido o interesse do meio acadêmico, por meio de trabalhos e referências. As Figuras de 7 a 12 apresentam a quantidade de trabalhos e citações referentes a essas metodologias. Pode-se inferir, nesses casos, que o interesse decorre do fato de que essas metodologias referem-se a assuntos mais específicos e atuais no contexto da Engenharia de Software. É possível observar o quantitativo crescente de citações da metodologia Service-oriented.

Figura 7 - Evolução de trabalhos Business Process Qtd trabalhos - Abordagem Business process

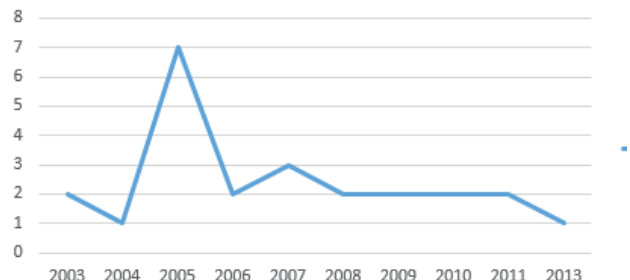

Fonte: do Autor

Figura 8 - Evolução citação Business Process Qtd citações - Abordagem Business process

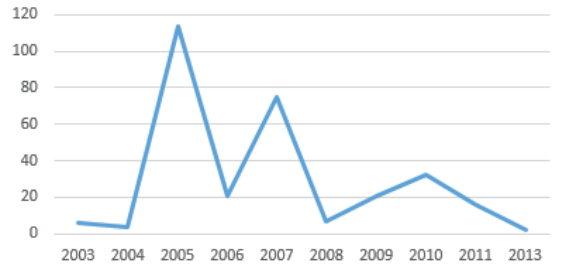

Fonte: do Autor

Figura 9 -. Evolução de trabalhos Agile Qtd trabalhos - abordagem Agile

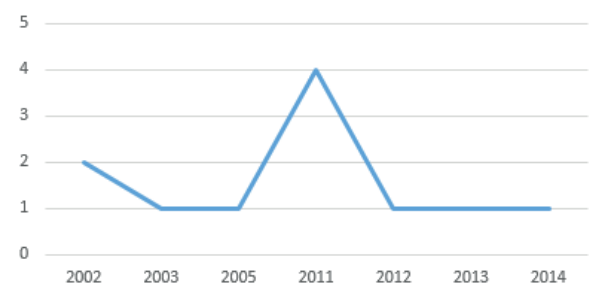

Fonte: do Autor

Figura 10 - Evolução citação Agile

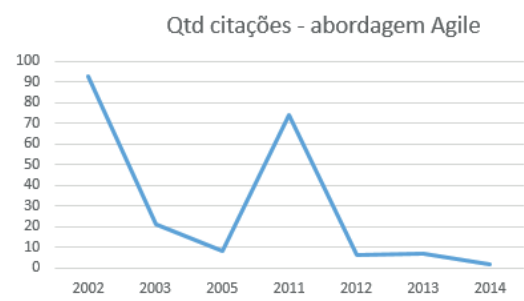

Fonte: do Autor

Figura 11 - Evolução de trabalhos Service-oriented Qtd trabalhos - Abordagem Service-Oriented

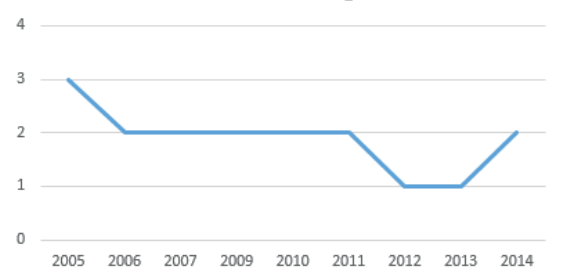

Fonte: do Autor 


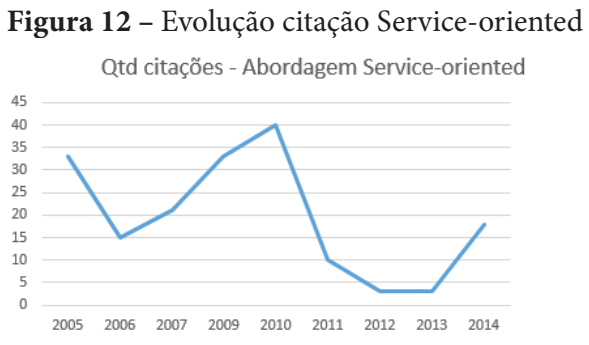

Fonte: do Autor

\section{Conclusões e trabalhos futuros}

Este artigo descreveu uma revisão sistemática sobre as metodologias existentes no domínio da Engenharia de Requisitos e suas experimentações. O objetivo da revisão foi identificar quais as metodologias da Engenharia de requisitos são mais citadas e/ou discutidas no meio acadêmico ou na industria identificando as implementações existentes e os tipos de pesquisa mais utilizados.

Para este trabalho, foram utilizadas as bases da Web of Science e o Google Acadêmico, considerando o período de 20 anos (1994 a 2014). Foram definidos o escopo da investigação, as perguntas da pesquisa, as palavras-chaves, critérios de seleção e exclusão de trabalhos. Foram elaboradas questões para avaliar a qualidade e adequação dos trabalhos ao contexto da pesquisa.

Foram analisados detalhadamente 184 artigos e identificaram-se as metodologias Goal, Scenário e Object-oriented como as mais representativas em quantitativo de artigos e citações.

Para responder as questões de pesquisa foram identificados que:

- Somente metade dos trabalhos possui experimentação e que destas a maior parte é no contexto da indústria, normalmente por meio de 1 estudo de caso;

- A maioria dos estudos estão baseados em evidências que foram apresentadas e complementadas com simulações.

As metodologias Business process, Agile e Service-Oriented, apesar de possuírem um quantitativo menor de citações, ainda estão sendo investigadas no meio acadêmico considerando o quantitativo de trabalhos e de citações nos últimos 5 anos.

A análise do quantitativo de trabalhos e citações permite inferir que as três metodologias citadas Goal, Scenario e Oriented-object estão consolidadas no meio acadêmico. Ainda existe espaço para experimentações, principalmente na indústria, dessas metodologias e das abordagens Business process, Agile e Service-Oriented.

\section{Referências}

ALLENBY, K.; KELLY, T. Deriving safety requirements using scenarios. In: IEEE INTERNATIONAL SYMPOSIUM ON REQUIREMENTS ENGINEERING, 5., 2001, Toronto. Proceedings... Washington: IEEE, 2001. p. 228-235.

ANTONELLI, L.; OLIVEROS, A. Fuentes y técnicas para elicitación de requerimientos: un estudio de evolución de la práctica. In: WORKSHOP DE INGENIERÍA DE REQUERIMIENTOS, 15., 2015, Lima. Anais... Lima, 2015. Disponible en: <http://eventos.spc.org.pe/cibse2015/ pdfs/04_WER15.pdf>. Acceso en: 12 abr. 2015.

BARROS, Aildil J.; LEHFELD, Neide A. Fundamentos de metodología científica. 3. ed. São Paulo: Pearson Prentice Hall, 2007.

BIOLCHINI, J.et al. Systematic review in software engeneering. Rio de Janeiro: PESC, 2005. (Technical Report RT ES 679/05)

CASTRO, J.; KOLP, M.; MYLOPOULOS, J. Towards requirements-driven information systems engineering: the Tropos project. Information systems, United Kingdom, v. 27, n. 6, p. 365-389, Sept. 2002.

DYBA, T.; DINGSOYR, T. Empirical studies of agile software development: a systematic review. Information and Software Technology, Massachusetts, v. 50, n. 9-10, p. 833-859, 2008.

ESPADA, P.; GOULÃO, M.; ARAÚJO, J. Measuring complexity and completeness of KAOS goal models. In: INTERNATIONAL WORKSHOP ON EMPIRICAL REQUIREMENTS ENGINEERING (EMPIRE2011), 2011, Trento. Proceedings... Washington: IEEE, 2011. p. 29-32.

EVERMANN, J.; WAND, Y. Ontology based object-oriented domain modelling: fundamental concepts. Requirements engineering, London, v. 10, n. 2, p. 146-160, May 2005.

FANTECHI, A.; GNESI, S.; LAMI, G.; MACCARI, A. Applications of linguistic techniques for use case analysis. Requirements Engineering, London, v. 8, n. 3, p. 161-170, 2003. 
FUXMAN, A. et al. Specifying and analyzing early requirements in Tropos. Requirements Engineering, London, v. 9, n. 2, p. 132-150, 2004.

GARCIA, C.; RAMIREZ, C. El meta análisis como instrumento de investigación en la determinacion y análisis del objeto del estudio: aplicado al estudio de sistema de información. In: CONGRESO INTERNACIONAL, 6.; CONGRESO IBEROAMERICANO DE DERECHO ROMANO, 9., 2004, Alicante. Actas... Alicante, 2004. p. $1-13$.

HARZING. Research in international management. Disponível em: <http://www.harzing.com/pop.htm>. Acesso em: 20 jul. 2015.

INSTITUTE OF ELECTRICAL AND ELECTRONICS ENGINEERS. IEEE Std 830: guide to software. Washington: IEEE, 1998.

JARKE, M.; BUI, X.; CARROLL, J. Scenario management: an interdisciplinary approach. Requirements Engineering, London, v. 3, n. 3-4, p. 155-173, 1998.

KAINDL, $\mathrm{H}$. Is object-oriented requirements engineering of interest? Requirements Engineering, London, v. 10, n. 1, p. 81-84, 2005.

KAIYA, H.; HORAI, H.; SAEKI, M. AGORA: Attributed goal-oriented requirements analysis method, 2002, Essen. In: IEEE JOINT INTERNATIONAL CONFERENCE ON REQUIREMENTS ENGINEERING. Proceedings... Washington: IEEE, 2003. p. 13-22.

KAVAKLI, E. Goal-oriented requirements engineering: a unifying framework. Requirements Engineering, London, v. 6, n. 4, p. 237-251, Jan. 2002.

KITCHENHAM, B. Charters: guidelines for performing systematic literature reviews in software engineering. Keele: Software Engineering Group, 2007.

LEITE, J. C.; HADAD, G.; KAPLAN, G. A scenario construction process. Requirements Engineering, London, v. 5, n. 1, p. 38-61, 2000.

LEITE, J. C. et al. Enhancing a requirements baseline with scenarios. Requirements Engineering, London, v. 2, n. 4, p. 184-198, 1997.
MAIDEN, N.; et al. CREWS-SAVRE: Systematic scenario generation and use. In: INTERNATIONAL CONFERENCE ON REQUIREMENTS ENGINEERING, 3., 1998, Colorado. Proceedings... Washington: IEEE, 1998. p. 1-31.

MUGNAINI, R.; STREHL, L. Recuperação e impacto da produção científica na era google: uma análise comparativa entre o google acadêmico e a web of science. Encontros Bibli: revista eletrônica de biblioteconomia e ciência da informação, Florianópolis, v. 13, n. 1, p. 92-105, 2008.

OLIVEIRA, K. et al. 25 years of Requirements Engineering in Brazil: a systematic mapping. WER, Montevideo, p. 1-17, 2013.

PEREZ-WILSON, M. Seis Sigma: compreendendo o conceito, as implicações e os desafios. Rio de Janeiro: Qualitymark, 1999.

ROLLAND, C. et al. A proposal for a scenario classification framework. Requirements Engineering, London, v. 3, n. 1, p. 23-47,1998.

ROLLAND, C.; SOUVEYET, C.; ACHOUR, C. Guiding goal modeling using scenarios. IEEE Transactions on Software Engineering, Washington, v. 24, n. 12, p. 1055-1071, 1998.

SANTANDER, V. F.; CASTRO, J. F. Deriving use cases from organizational modeling. IEEE JOINT INTERNATIONAL CONFERENCE ON REQUIREMENTS ENGINEERING, 2002, Essen. Proceedings... Washington, 2002. p. 32-39.

SOMMERVILLE, I.; SAWYER, P. Requirement Engineering: a good practice guide. Washington: John Wiley \& Sons, 1997.

SUTCLIFFE, A. G.; RYAN, M. Experience with SCRAM, a scenario requirements analysis method. In: INTERNATIONAL CONFERENCE ON REQUIREMENTS ENGINEERING, 3., 1998, Colorado. Proceedings... Washington: IEEE, 1998. p. 164-171.

SUTCLIFFE, A. G. et al. Supporting scenario-based requirements engineering. IEEE Transactions on Software Engineering, Washington, v. 24, n. 12, p. 1072-1088, 1998. 
SUTCLIFFE, A.; GAULT, B., MAIDEN, N. ISRE: immersive scenario-based requirements engineering with virtual prototypes. Requirements Engineering, London, v. 10, n. 2, p. 95-111, 2005.

VALASKI, J. et al. WER overview: retrospective, trends and relevance. CLEI Electronic Journal, Montevideo, v. 17, n. 3, 2014 .

VAN LAMSWEERDE, A. Goal-oriented requirements engineering: a guided tour. IEEE INTERNATIONAL SYMPOSIUM ON. 5., 2001, Toronto. Proceedings... Washington: IEEE, 2001. p. 249-262.

VAN LAMSWEERDE, A.; LETIER, E. Handling obstacles in goal-oriented requirements engineering. IEEE Transactions on Software Engineering, Washington, v. 26, n. 10 , p. $978-1005,2000$.
VAN LANSWEERDE, A. Goal-oriented requirements enginering: a roundtrip from research to practice [enginering read engineering]. In: IEEE INTERNATIONAL REQUIREMENTS ENGINEERING CONFERENCE, 12., 2004, Kyoto. Proceedings... Washington: IEEE, 2004. p. 4-7.

WEB OF SCIENCE. Thomson Reuters. Disponível em: $<$ http://www.webofknowledge.com>. Acesso em: 12 abr. 2015.

YU, E.; MYLOPOULOS, J. Why goal-oriented requirements engineering. INTERNATIONAL WORKSHOP ON REQUIREMENTS ENGINEERING, 4., 1998, Toronto. Proceedings... Toronto: Foundations of Software Quality, 1998. p. 15-22. 